\title{
COVID-19 and coagulative axis: review of emerging aspects in a novel disease
}

\author{
Matilde Boccia $^{1}$, Luigi Aronne ${ }^{1}$, Benito Celia ${ }^{1}$, Grazia Mazzeo ${ }^{1}$, Maria Ceparano1, Vito D’Agnano¹, \\ Roberto Parrella ${ }^{2}$, Tullio Valente ${ }^{3}$, Andrea Bianco ${ }^{1}$, Fabio Perrotta ${ }^{4}$ \\ ${ }^{1}$ Department of Translational Medical Sciences, University of Campania "L. Vanvitelli", Naples; ${ }^{2 D e p a r t m e n t ~ o f ~}$ \\ Infectious Diseases, COVID Unit D, Cotugno Hospital, A.O.R.N. dei Colli, Naples; ${ }^{3}$ Department of Radiology, Monaldi \\ Hospital, A.O.R.N. dei Colli, Naples; ${ }^{4}$ Department of Medicine and Health Sciences "V. Tiberio", University of Molise, \\ Campobasso, Italy
}

\begin{abstract}
Latest evidences from literature suggest that SARS-CoV-2 disease 2019 (COVID-19) is commonly complicated with coagulopathy and that disseminated intravascular coagulation is present in the majority of deceased patients. Particularly, conventional coagulation parameters appear to be significantly altered in patients with poor prognosis. A wide-ranging cross- talk between coagulative haemostasis and inflammation, as well as the activation of coagulation cascade during viral infections, are well established. Another important evidence which may explain coagulation disorders in COVID-19 is the increase of thrombus formation under conditions of hypoxia. Despite the exact pathophysiological mechanism of coronavirus-induced thromboembolism needs to be further investigated, this finding suggests that it is good practice to assess the risk of thrombosis in COVID-19 patients to improve the clinical management in terms of anticoagulation therapy. Anticoagulants, mainly low-molecularweight heparin (LMWH), should be tailored in patients meeting sepsis induced coagulopathy (SIC) criteria or with markedly elevated D-dimer. In this context, further studies are needed to optimise the decision making in therapeutic approach.
\end{abstract}

Correspondence: Prof. Fabio Perrotta, Department of Medicine and Health Sciences "V. Tiberio", University of Molise, 86100 Campobasso, Italy. E-mail: fabio.perrotta@unimol.it

Key words: COVID-19; SARS-CoV-2; coagulopathy; sepsis, disseminated intravascular coagulation.

Contributions: All the authors made a substantive intellectual contribution, have read and approved the final version of the manuscript and agreed to be accountable for all aspects of the work. MB and LA contributed equally.

Received for publication: 3 May 2020.

Accepted for publication: 12 May 2020.

${ }^{\circ}$ Copyright: the Author(s), 2020

Licensee PAGEPress, Italy

Monaldi Archives for Chest Disease 2020; 90:1300

doi: 10.4081/monaldi.2020.1300

This article is distributed under the terms of the Creative Commons Attribution Noncommercial License (by-nc 4.0) which permits any noncommercial use, distribution, and reproduction in any medium, provided the original author(s) and source are credited.

\section{SARS-CoV-2 infection breakthrough}

Since December, 2019, the world is experiencing an outbreak of a novel beta-coronavirus known as Severe Acute Respiratory Syndrome Coronavirus 2 (SARS- CoV-2). As of the $11^{\text {th }}$ of March, 2020, WHO has declared 2019 novel coronavirus disease (COVID-19) a pandemic infection [1].

Italy has been the first country after China to identify a significant outbreak of COVID-19 disease, with 212,532 confirmed cases and 27,402 associated deaths in the last update ( $6^{\text {th }}$ May 2020) [2]. COVID-19 patients who have died had a mean age of 79 years and an average of 3.3 co-morbidities, with cardiovascular diseases being the most common: $3.8 \%$ of deceased patients had no concomitant disease [3]. Growing evidence suggest that severe coronavirus disease 2019 is commonly complicated with coagulopathy and markedly elevated D-dimer was associated with poor prognosis of severe COVID-19 infection [4,5]. Recent prospective autoptic cohort study documented unpredictably elevated incidence of deep vein thrombosis (DVT) among deceased COVID-19 patients; massive pulmonary embolism (PE) was the direct cause of death in $33.3 \%$ of cases [6].

However, at this time comprehensive analysis of mechanisms underlying coagulative homeostasis unbalance are not fully depicted. We review the most recent evidences on interaction between pulmonary vascular endothelium and coagulative system implicated in pathogenetic mechanisms associated with SARS-CoV-2 infection.

\section{Emerging clinical data from COVID-19 cohorts}

Latest evidences from literature [7,8] suggest that COVID-19 is commonly complicated with coagulopathy and that disseminated intravascular coagulation (DIC) may exist in consistent number of deceased patients. DVT, PE or micro-thrombosis represent recurrent autoptic findings among COVID-19 patients [6,9]. In a recent clinical study, Han et al. [4] compared coagulation parameters including activated partial thromboplastin time (APTT), antithrombin (AT), fibrin/fibrinogen degradation products (FDP), fibrinogen (FIB), prothrombin time (PT), international normalized ratio (INR), prothrombin time activity (PT-act), and thrombin time (TT) between 94 patients with confirmed SARS-CoV-2 infection and 40 healthy controls. Compared with healthy controls, the AT values were found to be lower in COVID-19 patients $(85 \%$ vs $99 \% ; \mathrm{p}<0.001)$. The PT-act was also found to be lower in patients than in controls $(81 \%$ vs $97 \% ; \mathrm{p}<0.001)$, whereas the values of Ddimer (10.36 vs $0.26 \mathrm{ng} / \mathrm{L}$; p $<0.001)$, FDP (33.83 vs $1.55 \mathrm{mg} / \mathrm{L}$; $\mathrm{p}<0.001)$ and FIB (5.02 vs $2.90 \mathrm{~g} / \mathrm{L} ; \mathrm{p}<0.001)$ were higher in 
patients than in controls. No statistically significant differences were observed in values of APTT, PT, PT-INR, and TT between the two groups $(p>0.05)$. Interestingly, the entire cohort of SARSCoV-2 patients was divided into three groups, exhibiting an ordinary, severe, or critical clinical behaviour. No significant difference could be observed for APTT, PT, and PT-INR between three subgroups and the control group $(\mathrm{p}>0.05)$. However, D-dimer levels significantly differ between ordinary and severe disease groups ( $2.14 \pm 2.88$ vs $19.11 \pm 35.48 \mathrm{mg} / \mathrm{L} ; \mathrm{p}<0.01)$. These findings reflect a previous investigation [5], where coagulation parameters between survivors and non-survivors to novel coronavirus pneumonia (NCP) were compared on admission and at three-days intervals from day 1 to day 14 after admission. The non-survivors revealed significantly higher D-dimer and FDP levels, and longer PT compared to survivors on admission. By the late hospitalization, the FIB and AT levels were significantly lower in non-survivors. Besides, during NCP late stages, the levels of fibrin-related markers (D-dimer and FDP) appeared to be moderately or markedly elevated in all deaths. These findings reflect a coagulation activation and a secondary hyperfibrinolysis, as expression of DIC. According to the International Society on Thrombosis and Haemostasis (ISTH) diagnostic criteria for DIC, 15 (71.4\%) of the non-survivors matched the grade of overt-DIC ( $\geq 5$ points) in later stages of NCP. The median time from admission to DIC was 4 days (range: 1-12 days). Conversely, only one (0.6\%) survivor matched the DIC criteria during hospital stay. Likewise, mild thrombocytopenia is associated with a higher risk of requiring mechanical ventilation, intensive care unit [ICU] admission, or death related to COVID-19 [10]. Klok et al. evaluated the incidence of the composite outcome of venous thromboembolism (VTE) and arterial thrombotic complications in 184 patients with proven COVID-19 pneumonia admitted to the ICU in between March $7^{\text {th }}$ and April $5^{\text {th }}$ 2020. Composite outcome consisted of acute PE, DVT, ischemic stroke, myocardial infarction or systemic arterial embolism. Patients were followed until ICU discharge/death or time deadline. The cumulative incidence of the composite outcome was 31\%. PE was the most frequent thrombotic complication $(n=25,81 \%)$. Notably, all patients received at least standard doses thromboprophylaxis, although regimens differed between hospitals [11]. Additionally, in another single-centre study, Cui et al. reported the prevalence of VTE in 81 severe COVID-19 patients admitted to the ICU without preventive anticoagulant administration. VTE incidence was $25 \%(20 / 81)$, resulting in fatal death in $40 \%(8 / 20)$ of cases. Increased levels of d-dimer $>1.5 \mathrm{mg} / \mathrm{ml}$ (normal range: $0.0-0.5 \mathrm{mg} / \mathrm{ml}$ ) predicted VTE with sensitivity $85 \%$, specificity $88.5 \%$ and negative predictive value $94.7 \%$ [12].

In addition, increased mortality in COVID-19 cohorts has been reported among patients with active cancer [7,13,14]. Complex mechanisms regulate cancer associated thrombosis promoting both oncologic progression, immune system regulation and in vascular clot formation [15-19]. Cancer cells aberrantly produce tissue factor (TF) resulting in extrinsic coagulation pathway activation and consequently in fibrin synthesis and platelet activation. Such evidences suggested that conventional coagulation parameters during the course of NCP were significantly associated with prognosis.

\section{Virus induced inflammation and coagulative homeostasis: a complex cross-talk}

Complex wide-ranging cross-talk between coagulative haemostasis and inflammation during viral pneumonia has been postulated [20-22]. Inflammatory mediators can elevate platelet count, platelet reactivity, down-regulate natural anticoagulant mechanisms, initiate the coagulation system, facilitate propagation of the coagulant response and impair fibrinolysis [23-28].

\section{Pathogenetic mechanisms of exalted thrombophilic risk during viral pneumonia: what we have learned from SARS-CoV}

Evidence from previous epidemic outbreaks documented that in patients with SARS-CoV a panel of genes with procoagulant effect was highly expressed within infected mononuclear cells: these include fibrinogen (FIB), SERPINs, factors II, III and X, thromboxane synthase (TBXAS) and Toll-like receptor 9 (TLR9) $[29,30]$. Molecular mechanisms of exalted thrombophilic risk during SARS-CoV pneumonia also comprise peripheral vasoconstriction induced from the exalted thromboxane production promotes, platelet aggregation, and endothelial dysfunction [31]. The TLR9 is expressed in platelets and - upon ligand binding promotes platelet activation, degranulation, and aggregation [32]. Furthermore, SARS-CoV nucleocapsid protein has also been considered as one of the important determinants of SARS prothrombotic state, inducing the human fibrinogen-like protein-2 (HFGL2) prothrombinase gene [33]. Dysregulation of the urokinase pathway and other associated prothrombotic genes have also been implicated in the pathogenesis of SARS-CoV related coagulation disorders. Evidence from SARS-CoV mouse model demonstrated that procoagulant genes expression (thrombin, VII, XI, XII), plasminogen activators expression and urokinase pathway dysregulation have been associated with fatal SARS-CoV infection [34]. SARS-CoV and SARS-CoV-2 share up to $85 \%$ genomic identity, and both utilize the same primary human host receptor, angiotensin converting enzyme 2 (ACE2), to enter target cells [35]. Due to their homology, similar clinical and immunopathologic aspects are expected to occur, but differences may also exist, and caution is needed when extrapolating data from SARS to COVID-19.

In both SARS and COVID-19 patients a derangement of reninangiotensin-aldosterone system (RAAS) is observed, that is intrinsically linked to the coagulation cascade and may exacerbate the process of immune-thrombosis, further driving microthrombi formation in COVID-19 [36]. Firstly, angiotensin II (AngII) induces plasminogen activator inhibitor type 1 (PAI-1) expression by endothelial cells via the AT1 receptor, contributing to a PAI-1/tPA imbalance and a hypercoagulable state [37]. Interestingly, AngII also stimulates PAI-1 release from adipocytes via AT1 receptors, and may in part account for the increased severity observed in those with high BMI [38]. Moreover, ACE metabolizes bradykinin, that finally stimulate vasodilation and release of PAI-1 from endothelial cells [39].

\section{Inflammatory factors promoting the coagulative axis disruption during acute viral infection}

Several inflammatory mediators are implicated in coagulative dysregulation during acute infectious event. Firstly, tissue factor (TF), the primary initiator of the blood coagulation cascade, that ensures rapid haemostasis in case of organ damage through its receptor activity for factor VII: inflammatory cytokines, such as tumor necrosis factor (TNF)- $\alpha$, endotoxin and interleukins strongly induce expression of TF on the cell surface of endothelium and leucocytes, particularly monocytes. TF promotes pleiotropic inflammatory responses but, alteration of its expression, gives a strong contribution to hypercoagulable state [40]. 
Secondly, FIB concentration, as acute phase protein, is a relevant mediator of the coagulative axis; increase in FIB levels have been associated with an increased risk of thrombotic disease [41] . Thirdly, histamine, TNF-a, IL-8 and IL-6 all lead to the release of ultra-large von Willebrand factor (vWF) multimers from the endothelium: these large multimers are particularly effective at promoting platelet aggregation. Normally, the high molecular weight multimers are processed by ADAMTS13 to generate less thrombogenic smaller forms: this process appears to be inhibited by IL-6 [41].

In acute inflammatory processes, such as sepsis, antithrombin is consumed and/or inactivated [41]. In addition, the concentration of vascular heparin-like molecules can be reduced by inflammatory cytokines and neutrophil activation products. Within natural anticoagulant pathways, the C-protein pathway is highly sensitive to downregulation induced by inflammatory mediators (Endotoxin, IL$1 \mathrm{~b}$, and TNF-a) [42]. Furthermore, fibrin degradation is severely impaired by the inactivation of the endogenous fibrinolytic system, mainly as a result of upregulation of its principal inhibitor, PAI-1 [43]. Finally, elevated D-dimer reported in patients with severe COVID-19 are suspected to reflect the significant imbalance in thrombin generation and fibrinolysis, especially in the pulmonary vasculature. In both general ARDS and sepsis, significant evidence supports a procoagulant state characterized by massive thrombin production. In SARS-CoV was shown to upregulate HFGL2 prothrombinase gene, which may possibly contribute to thrombin generation and hypercoagulable state in COVID-19 [31].

\section{Virus-induced endothelial dysfunction}

Viral infection may directly induce cytopathic endothelial cell damage. Relevant evidences documented that most of the common viruses (Herpes simplex, Adenovirus, Parainfluenza virus, Echovirus, Poliovirus, Measles, HIV, CMV, etc.) directly infect the endothelial cells.

Since vascular endothelium actively expresses ACE2, and was shown to be an active site of SARS-CoV infection [44], it is obviously plausible that SARS-CoV-2 is capable of directly infecting these cells [45]. This results in increased vascular permeability, excess thrombin generation (also a major activator of platelets) and inhibition of fibrinolysis [46]. Furthermore, it has been postulated that the increased risk of severe and fatal COVID-19 in patients with comorbidities such as diabetes, hypertension, and obesity may in part be due to the underlying endothelial dysfunction, which is common in these conditions [36].

\section{Complement activation in viral pneumonia}

A mouse model of SARS demonstrates that dysregulated complement activation contributes to coagulopathy [47]: bioactive complement molecules, such as C3a, C5a, membrane attack complex (MAC), not only exert a multitude of pro-inflammatory effects, including mast cell degranulation and leucocyte recruitment, but they also exert pro-thrombotic effects through activation of platelets and endothelial cells, as well as increasing tissue factor and von Willebrand factor (VWF) expression. Other proteins of the complement system (MASP-1 and MASP-2) cooperate to the generation of hypercoagulable state by converting prothrombin to thrombin and fibrinogen to fibrin [48]. A pattern of tissue damage consistent with complement-mediated microvascular injury has been noted in the lung and/or skin of five individuals with severe COVID-19: the deposition of C5b-9, C4d, and MASP2 in the microvasculature of the two organs is consistent with profound and generalized activation of both alternative and lectin-based path- ways. This provides a foundation for further exploration of the pathophysiologic importance of complement in COVID-19 [49].

\section{Other potential factors contributing to hypercoagulable state in COVID-19}

The hypercoagulable state may potentially be further enhanced by other clinical factors including hypoxemia (secondary to ALI/ARDS), hyperthermia (which may activate platelets and coagulation), and/or hypovolemia (secondary to gastrointestinal fluid loss and/or negative fluid balance in the ARDS treatment protocol) [36]. Vascular response to hypoxia is controlled primarily by the hypoxia-inducible transcription factors (HIFs); HIFs may promote further inflammation, thus augmenting blood viscosity and contributing to worsen hypercoagulability. Moreover, HIFs may directly activate platelets and coagulation factors, increasing TF expression, increasing PAI-1, and inhibiting the endogenous anticoagulant protein [27]. A recent study reported 3 cases with severe COVID-19 and cerebral infarction in the setting of elevated antiphospholipid antibodies: whether antiphospholipid antibodies play a major role in pathophysiology of thrombosis associated with COVID-19 requires further investigation [50].

\section{Coagulative unbalance in COVID-19: implications for clinical and therapeutic management}

The coagulation disorder found in COVID-19 patients may influence the clinical management in terms of decision making for therapy. Analogously to other acute medical patients, VTE risk stratification for hospitalized patients with COVID-19 should be considered. Multiple risk stratification tools are currently available for VTE risk assessment (e.g., the Caprini, IMPROVE, and Padua models) [51]. A recent study reports that $40 \%$ of hospitalized patients with COVID-19 were at high risk of VTE based on Padua model [52]. The World Health Organization interim guidance statement recommends prophylactic daily low-molecular weight heparins (LMWHs), or twice daily subcutaneous unfractionated heparin (UFH) in patients with suspected COVID-19 pneumonia [53]. When pharmacological prophylaxis is contraindicated, mechanical VTE prophylaxis (intermittent pneumatic compression) should be considered in immobilized patients [53,54]. In this regard, once daily dosing regimen of LMWHs may be advantageous over UFH to reduce personal protective equipment (PPE) use and exposure of healthcare workers [55].

In a retrospective single-center study, Tang et al. [56] analysed four hundred and forty-nine patients (181 females and 268 males) classified as severe COVID-19 from consecutive 1786 confirmed cases, comparing the 28-day mortality between heparin users [ninety-nine of which 94 received low molecular weight heparin, LMWH (40-60 mg enoxaparin/day) and 5 received un-fractioned heparin, UFH (10000-15000 U/day), for 7 days or longer] and nonusers. The 28-day mortality was also evaluated according to the different risk of coagulopathy which was stratified by the sepsisinduced coagulopathy (SIC) score or D-dimer result. No difference on the 28-day mortality was found between heparin users and nonusers $(30.3 \%$ vs $29.7 \%, \mathrm{p}=0.910)$. However, the heparin treatment was associated with lower mortality in patients with SIC score $\geq 4$ (40.0\% vs 64.2\%, $\mathrm{p}=0.029)$, but not in those with SIC score $<4$ (29.0\% vs $22.6 \%, \mathrm{p}=0.419)$. When patients were stratified according to D-dimer levels, the mortality in heparin users basically remained at same level, but in non-users the mortality raised pro- 
portionally with D-dimer levels. When D-dimer exceeded 3.0 $\mu \mathrm{g} / \mathrm{ml}$ (6-fold of upper limit of normal), approximately $20 \%$ reduction in mortality with heparin treatment was found $(32.8 \% \mathrm{vs}$ $52.4 \%, \mathrm{p}=0.017)$. Since the activation of coagulation also contributes to compartmentalization of pathogens and reduces their invasion [57], anticoagulant treatment in patients without significant coagulopathy has a potential risk. Therefore, anticoagulants should not be extended to every patient with COVID-19 but only to those meeting SIC criteria or with markedly elevated D-dimer, mainly LMWH. In such patients the use of LMWH could be useful in preventing DIC also based on anti-inflammatory effects. Firstly, heparin inhibits the activation and function of neutrophils. Secondly, heparin counteracts the expression of inflammatory mediators which initiate and drive activation of the innate immune system through reducing the translocation of the transcription factor nuclear factor-kappaB $(\mathrm{NF}-\kappa \mathrm{B})$ from the cytoplasm into the nucleus; likewise, reduced levels of TNF-alpha, IL-6, IL-8, and IL1 beta are promoted. While multiple studies have demonstrated the anti-inflammatory effects of the heparins, less is known about the differences among various heparin fractions and anti-inflammatory actions [58]. Direct oral anticoagulants (DOACs) plasmatic concentration in COVID-19 patients treated with antiviral drugs showed an alarming increase (6.14 times higher than in the prehospitalization period). Based on this finding, current evidence in SARS-CoV-2 patients suggest DOACs replacement with alternative parenteral antithrombotic strategies for as long as antiviral agents are deemed necessary and/or until discharge [59].

After hospital discharge, individualized risk stratification for thrombotic and haemorrhagic risk need to be assessed. Extended VTE prophylaxis (for up to 45 days) would be recommended in patients with elevated VTE risk (e.g., reduced mobility, co-morbidities such as active cancer and, according to some authors, elevated $\mathrm{D}$-dimer $>2$ times the upper normal limit) with low bleeding risk [60,61]; unfortunately, at this time no specific guidelines for COVID-19 patients are available.

Finally, glucocorticoids are widely used in COVID-19 though several clinical trials are still ongoing. They counterbalance the inflammation though potentially prompting coagulation system activation. Indeed, levels of vWF, factor VII, factor VIII, factor XI and FBG are increased and the platelet count was found to be risen in patients using therapeutic doses of dexamethasone possibly resulting in exalted thrombotic risk [62]. Based on above studies, targeting the complex network between coagulative axis and inflammation would result in beneficial outcomes for patients with COVID-19; unfortunately, the magnitude of anticoagulants and glucocorticoids pharmacological effects at this stage is weak and there is urgent need of more comprehensive cohort studies.

\section{Conclusions}

The emerging evidence coming from literature suggest that SARS-CoV-2 infection negatively affects coagulation system at different levels. Molecular trafficking among inflammatory mediators, coagulative factors, platelets and vascular endothelium comprises complex interactions far to be definitively dissected. Pharmacological treatment targeting coagulative axis and inflammation need stronger evidence, though LMWH could be beneficial even for the intrinsic anti-inflammatory effect. The exact pathophysiological mechanism of SARS-CoV-2 induced thromboembolism needs to be further studied to provide biological bases to improve clinical anticoagulation therapy.

\section{References}

1. WHO. WHO announces COVID-19 outbreak a pandemic. Available from: http:/www.euro.who.int/en/health-topics/ health-emergencies/coronavirus-covid-19/news/news/2020/ 3/who-announces-covid-19-outbreak-a-pandemic

2. Istituto Superiore di Sanità (ISS). Sorveglianza Integrata COVID-19 in Italia. Available from: https://www.epicentro. iss.it/coronavirus/bollettino/Infografica_6maggio\%20ITA.pdf

3. Istituto Superiore di Sanità (ISS). Caratteristiche dei pazienti deceduti positivi all'infezione da SARS-CoV-2 in Italia. Available from: https://www.epicentro.iss.it/coronavirus/bollettino/Report-COVID-2019_29_aprile.pdf

4. Han H, Yang L, Liu R, et al. Prominent changes in blood coagulation of patients with SARS-CoV-2 infection. Clin Chem Lab Med 2020. doi: 10.1515/cclm-2020-0188.

5. Tang N, Li D, Wang X, Sun Z. Abnormal coagulation parameters are associated with poor prognosis in patients with novel coronavirus pneumonia. J Thromb Haemost 2020. doi: 10.1111/jth.14768.

6. Wichmann D, Sperhake J-P, Lütgehetmann M, et al. Autopsy findings and venous thromboembolism in patients with COVID-19: A prospective cohort study. Ann Intern Med 2020. doi: 10.7326/M20-2003.

7. Huang C, Wang Y, Li X, et al. Clinical features of patients infected with 2019 novel coronavirus in Wuhan, China. Lancet 2020;395:497-506.

8. Chen N, Zhou M, Dong X, et al. Epidemiological and clinical characteristics of 99 cases of 2019 novel coronavirus pneumonia in Wuhan, China: a descriptive study. Lancet 2020;395: 507-13.

9. Yao XH, Li TY, He ZC, et al. [A pathological report of three COVID-19 cases by minimally invasive autopsies].[Article in Chinese]. Zhonghua bing li xue za zhi 2020;49:E009. doi: 10.3760/cma.j.cn112151-20200312-00193.

10. Lippi G, Plebani M, Henry BM. Thrombocytopenia is associated with severe coronavirus disease 2019 (COVID-19) infections: A meta-analysis. Clin Chim Acta 2020;506:145-8. doi: 10.1016/j.cca.2020.03.022.

11. Klok FA, Kruip MJHA, van der Meer NJM, et al. Incidence of thrombotic complications in critically ill ICU patients with COVID-19. Thromb Res 2020. doi: 10.1016/j.thromres.2020.04.013

12. Cui S, Chen S, Li X, et al. Prevalence of venous thromboembolism in patients with severe novel coronavirus pneumonia. J Thromb Haemost 2020. doi: 10.1111/jth.14830.

13. Zhang J-J, Dong X, Cao Y-Y, et al. Clinical characteristics of 140 patients infected with SARS-CoV-2 in Wuhan, China. Allergy 2020. doi: 10.1111/all.14238.

14. $\mathrm{Xu} \mathrm{Y,} \mathrm{Liu} \mathrm{H}, \mathrm{Hu} \mathrm{K}$, Wang M. [Clinical management of lung cancer patients during the outbreak of 2019 novel coronavirus disease (COVID-19)].[Article in Chinese]. Zhongguo Fei Ai Za Zhi 2020;23:136-41. doi: 10.3779/j.issn.1009-3419. 2020.03.02.

15. Fernandes CJ, Morinaga LTK, Alves JLJ, et al. Cancer-associated thrombosis: the when, how and why. Eur Respir Rev 2019;28. doi: 10.1183/16000617.0119-2018.

16. Nigro E, Stiuso P, Matera MG, et al. The anti-proliferative effects of adiponectin on human lung adenocarcinoma A549cells and oxidative stress involvement. Pulm Pharmacol Ther 2019. doi: 10.1016/j.pupt.2019.01.004.

17. Bianco A, Perrotta F, Barra G, Malapelle U, Rocco D, De 
Palma R. Prognostic factors and biomarkers of responses to immune checkpoint inhibitors in lung cancer. Int J Mol Sci 2019;20. doi: 10.3390/ijms20194931.

18. Bianco A, Malapelle U, Rocco D, et al. Targeting immune checkpoints in non small cell lung cancer. Curr Opin Pharmacol 2018;40:46-50.

19. Di Zazzo E, Polito R, Bartollino S, et al. Adiponectin as link factor between adipose tissue and cancer. Int J Mol Sci 2019;20. doi: 10.3390/ijms20040839.

20. Bianco A, Mazzarella G, Turchiarelli V, et al. Adiponectin: an attractive marker for metabolic disorders in chronic obstructive pulmonary disease (COPD). Nutrients 2013;5:4115-25.

21. Perrotta F, Nigro E, Mollica M, et al. Pulmonary hypertension and obesity: Focus on adiponectin. Int J Mol Sci 2019;20. doi: 10.3390/ijms20040912.

22. Schouten M, Wiersinga WJ, Levi M, van der Poll T. Inflammation, endothelium, and coagulation in sepsis. J Leukoc Biol 2008;83:536-45.

23. Esmon $\mathrm{CT}$. The interactions between inflammation and coagulation. Br J Haematol 2005;131:417-30.

24. Illiano M, Nigro E, Sapio L, et al. Adiponectin down-regulates CREB and inhibits proliferation of A549 lung cancer cells. Pulm Pharmacol Ther 2017;45:114-20.

25. Giannattasio A, Brunese L, Ripabelli G, et al. Coinfections with influenza virus and atypical bacteria: Implications for severe outcomes? Clin Respir J 2018;12:366-7.

26. Bianco A, Nigro E, Monaco ML, et al. The burden of obesity in asthma and COPD: Role of adiponectin. Pulm Pharmacol Ther 2017;43:20-5.

27. Maniscalco M, Bianco A, Mazzarella G, Motta A. Recent advances on nitric oxide in the upper airways. Curr Med Chem 2016;23:2736-45.

28. Maniscalco M, Vitale C, Vatrella A, et al. Fractional exhaled nitric oxide-measuring devices: technology update. Med Devices (Auckl) 2016;9:151-60.

29. Wong RSM, Wu A, To KF, et al. Haematological manifestations in patients with severe acute respiratory syndrome: Retrospective analysis. Br Med J 2003;326:1358-62.

30. Ng LFP, Hibberd ML, Ooi EE, et al. A human in vitro model system for investigating genome-wide host responses to SARS coronavirus infection. BMC Infect Dis 2004;4:1-11.

31. Ashton AW, Ware JA. Thromboxane A2 receptor signaling inhibits vascular endothelial growth factor-induced endothelial cell differentiation and migration. Circ Res 2004;95:372-9.

32. Panigrahi S, Ma Y, Hong L, et al. Engagement of platelet tolllike receptor 9 by novel endogenous ligands promotes platelet hyperreactivity and thrombosis. Circ Res 2013;112:103-12.

33. Han M, Yan W, Huang Y, et al. The nucleocapsid protein of SARS-CoV induces transcription of hfgl2 prothrombinase gene dependent on C/EBP alpha. J Biochem 2008;144:51-62.

34. Gralinski LE, Baric RS. Molecular pathology of emerging coronavirus infections. J Pathol 2015;235:185-95.

35. Perrotta F, Matera MG, Cazzola M, Bianco A. Severe respiratory SARS-CoV2 infection: Does ACE2 receptor matter? Respir Med 2020;168:105996.

36. Henry BM, Vikse J, Benoit S, et al. Hyperinflammation and derangement of renin-angiotensin-aldosterone system in COVID-19: A novel hypothesis for clinically suspected hypercoagulopathy and microvascular immunothrombosis. Clin Chim Acta 2020;507:167-73.

37. Vaughan DE, Lazos SA, Tong K. Angiotensin II regulates the expression of plasminogen activator inhibitor-1 in cultured endothelial cells: a potential link between the renin- angiotensin system and thrombosis. J Clin Invest 1995;95:9951001.

38. Skurk T, Lee YM, Hauner H. Angiotensin II and its metabolites stimulate PAI-1 protein release from human adipocytes in primary culture. Hypertension 2001;37:1336-40.

39. Brown NJ, Nadeau JH, Vaughan DE. Selective stimulation of tissue-type plasminogen activator (t-PA) in vivo by infusion of bradykinin. Thromb Haemost 1997;77:522-5.

40. Witkowski M, Landmesser U, Rauch U. Tissue factor as a link between inflammation and coagulation. Trends Cardiovasc Med 2016;26:297-303.

41. Levi M, Poll T Van Der. Coagulation in patients with severe sepsis. Int J Cardiol 2011;148:276-9.

42. Levi M, van der Poll T. Coagulation and sepsis. Thromb Res 2017;149:38-44.

43. Schmitt FCF, Manolov V, Morgenstern J, et al. Acute fibrinolysis shutdown occurs early in septic shock and is associated with increased morbidity and mortality: results of an observational pilot study. Ann Intensive Care 2019;9:19.

44. Ye J, Zhang B, Xu J, et al. Molecular pathology in the lungs of severe acute respiratory syndrome patients. Am J Pathol 2007;170:538-45.

45. Nicin L, Abplanalp WT, Mellentin H, et al. Cell type-specific expression of the putative SARS-CoV-2 receptor ACE2 in human hearts. Eur Heart J 2020. doi: 10.1093/eurheartj/ehaa311.

46. Frantzeskaki F, Armaganidis A, Orfanos SE. Immunothrombosis in acute respiratory distress syndrome: cross talks between inflammation and coagulation. Respiration 2017;93:212-25

47. Gralinski LE, Sheahan TP, Morrison TE, et al. Complement activation contributes to severe acute respiratory syndrome coronavirus pathogenesis. MBio 2018;9:1-15.

48. Keragala CB, Draxler DF, McQuilten ZK, Medcalf RL. Haemostasis and innate immunity - a complementary relationship: A review of the intricate relationship between coagulation and complement pathways. Br J Haematol 2018;180:782-98.

49. Magro C, Mulvey JJ, Berlin D, et al. Complement associated microvascular injury and thrombosis in the pathogenesis of severe COVID-19 infection: a report of five cases. Transl Res 2020. doi: 10.1016/j.trsl.2020.04.007.

50. Zhang Y, Xiao M, Zhang S, et al. Correspondence Coagulopathy and antiphospholipid antibodies in patients with Covid-19. N Engl J Med 2020;382:e38.

51. Liu X, Liu C, Chen X, et al. Comparison between Caprini and Padua risk assessment models for hospitalized medical patients at risk for venous thromboembolism: A retrospective study. Interact Cardiovasc Thorac Surg 2016;23:538-43.

52. Wang T, Chen R, Liu C, et al. Attention should be paid to venous thromboembolism prophylaxis in the management of COVID-19. Lancet Haematol 2020;7:e362-3.

53. WHO. Clinical management of severe acute respiratory infection (SARI) when COVID-19 disease is suspected. Available from: https://www.who.int/publications-detail/clinical-management-of-severe-acute-respiratory-infection-when-novelcoronavirus-(ncov)-infection-is-suspected

54. Ho KM, Tan JA. Stratified meta-analysis of intermittent pneumatic compression of the lower limbs to prevent venous thromboembolism in hospitalized patients. Circulation 2013;128:1003-20.

55. Bikdeli B, Madhavan MV, Jimenez D, Cet al. COVID-19 and thrombotic or thromboembolic disease: implications for prevention, antithrombotic therapy, and follow-up. J Am Coll Cardiol 2020. doi: 10.1016/j.jacc.2020.04.031. 
56. Tang N, Bai H, Chen X, et al. Anticoagulant treatment is associated with decreased mortality in severe coronavirus disease 2019 patients with coagulopathy. J Thromb Haemost 2020. doi: $10.1111 /$ jth. 14817 .

57. Sun H, Wang X, Degen JL, Ginsburg D. Reduced thrombin generation increases host susceptibility to group A streptococcal infection. Blood 2009;113:1358-64.

58. Poterucha TJ, Libby P, Goldhaber SZ. More than an anticoagulant: Do heparins have direct anti-inflammatory effects? Thromb Haemost 2017;117:437-44.

59. Testa S, Prandoni P, Paoletti O, et al. Direct oral anticoagulant plasma levels striking increase in severe COVID-19 respirato- ry syndrome patients treated with antiviral agents. The Cremona experience. J Thromb Haemost 2020:1-4.

60. Cohen AT, Spiro TE, Spyropoulos AC, et al. D-dimer as a predictor of venous thromboembolism in acutely ill, hospitalized patients: A subanalysis of the randomized controlled MAGELLAN trial. J Thromb Haemost 2014;12:479-87.

61. Schindewolf M, Weitz JI. Broadening the categories of patients eligible for extended venous thromboembolism treatment. Thromb Haemost 2020;120:14-26.

62. Isidori AM, Minnetti M, Sbardella E, et al. The spectrum of haemostatic abnormalities in glucocorticoid excess and defect. Eur J Endocrinol 2015;173:R101-13. 